\title{
Programa para el Desarrollo Emocional de los Niños Preescolares
}

Mariluces Amaya de Lee, Beatriz Restrepo de Franco, Carmen Elisa de Prías, Ana Rosalba Lizarazo de Nempeque, Martha Tobón de Awad.

Por un Mañana Santa Fe de Bogotá, Colombia.

La frecuente situación de egocentrismo, la falta de valores y violencia, que se vive en algunos lugares del mundo actual, ha llevado a varios autores a trabajar sobre la influencia que tiene el desarrollo emocional en la vida de las personas. En el Jardín Infantil Por un Mañana, se ha implementado un programa de desarrollo emocional, teniendo en cuenta la importancia del tema y la necesidad sentida de trabajarlo en nuestro país.

El programa se diseño siguiendo las pautas del Modelo de Pedagogía Participativa (Amaya, Lizarazo, y otros, 1997), el cuál se centra en el niño, aplicando acciones educativas que integran los saberes de la Pedagogía, la Terapia Ocupacional y la Fonoaudiología. El programa se propone para la etapa pre-escolar y por lo tanto las estrategias y actividades están dirigidas a niños entre uno y medio y cinco años. Sin embargo se considera que los planteamientos son válidos para niños mayores, en cualquier grado escolar.

Se ha fundamentado en los planteamientos de los siquiatras Luis Carlos Restrepo y Daniel Goleman, Las sicólogas Ana María León, Lucia Pinto y Martha Rodríguez. Sus objetivos son: Facilitar y hacer amable al niño, el paso de la casa al colegio; desarrollar el conocimiento personal; propiciar el conocimiento del otro; favorecer el sentido de grupo; y enseñar a actuar democráticamente.

Palabras claves: Desarrollo Emocional, expresión de emociones, valores humanos, autoconocimiento, autoestima, sentido de grupo y cooperación social. 
El objetivo de este artículo es presentar un programa que responde a la necesidad de trabajar el desarrollo emocional desde la primera infancia a través de un curriculo diseñado cuidadosamente.

Primero justifica el valor de tener una ciencia de desarrollo emocional en la etapa preescolar; luego se expone el marco teórico que lo sustenta. Posteriormente se hace la propuesta para implementar el programa. Finalmente se ejemplifican algunas estrategias a través de una experiencia en el Jardín Infantil Por un Mañana.

La propuesta de crear un curriculo de desarrollo emocional, se basa en la experiencia de las autoras durante trece años, en el trabajo cotidiano con niños preescolares. Además este forma parte de los programas pedagógicos del Jardín Infantil Por un Mañana, el cual desarrolla su actividad con el modelo de Pedagogía Participativa (Amaya, Lizarazo, otros 1996).

Los argumentos teóricos que se presentan en este artículo se basan en los planteamientos de los siguientes autores : Daniel Goleman, Luis Carlos Restrepo, Martha Rodríguez, Ana María León, Lucía Pinto, Lawrence Shapiro y Howard Gardner.

\section{Justificación}

La situación de egocentrismo, falta de valores y violencia, que se vive en el mundo actual, ha llevado a varios autores a trabajar sobre la influencia que tiene el desarrollo emocional en la vida de las personas. Con estos aportes, se han escrito y propuesto métodos para favorecer la formación ética y emocional en los niños, con la idea de desarrollar otra actitud personal la cual combata la situación que se vive hoy en día.

En el Jardín Infantil Por un Mañana, se ha implementado un programa de desarrollo emocional, teniendo en cuenta la importancia del tema y la necesidad sentida de trabajarlo en nuestro país.

El programa se propone para la etapa pre-escolar y por lo tanto las estrategias y actividades están dirigidas a niños entre uno y medio y cinco años. Sin embargo se considera que los planteamientos son válidos para niños mayores, en cualquier grado escolar. 
Para concebir el programa se tuvo en cuenta el proceso de desarrollo emocional de los niños desde la gestación hasta los seis años. Un résumen de los eventos más importantes que aprende el niño en este período se encuentra en el cuadro No. 1 que se encuentra à continuación.

Gráfica No. 1. PROCESO DE DESARROLLO EMOCIONAL HASTA LOS SEIS AÑOS.

\begin{tabular}{|c|c|c|c|}
\hline EDAD & $\mathrm{YO}$ & YOYEL OTRO & YO Y LOS DEMÁS \\
\hline GESTACION & $\begin{array}{l}\text { Uora y se sonrie. } \\
\text { Tiene formas de } \\
\text { comportarse y de } \\
\text { reaccionar ante diferentes } \\
\text { situaciones. }\end{array}$ & $\begin{array}{l}\text { Hace movimientos fuertes y } \\
\text { rapidos ante lo que vive la } \\
\text { madre. } \\
\text { Capta las emociories que } \\
\text { experimenta su madre. }\end{array}$ & 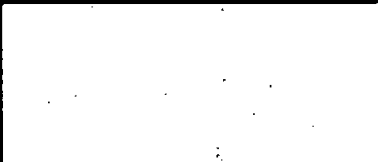 \\
\hline 0 alaño & $\begin{array}{l}\text { sonrie. } \\
\text { - Vocaliza sus estados } \\
\text { placenteros con gritos, } \\
\text { gorgeos y risitas. } \\
\text { Sonrie y vocaliza ante su } \\
\text { imagen en el espejo. } \\
\text { - Manifiesta sus estados de } \\
\text { animo : Furia, tristeza y } \\
\text { alegría. } \\
\text { Explora su cuerpo con } \\
\text { manos y boca. }\end{array}$ & $\begin{array}{l}\text { - Tiene una estrecha relación } \\
\text { - } \text { - Deja de ilorar cuando se le } \\
\text { toma en brazos } \\
\text { - Observa las caras } \\
\text { - Fija los ojos en las personas.. } \\
\text { Le gusta el contacto con } \\
\text { personas que le son } \\
\text { familiares. } \\
\text { - Goza cuando lo alzan y lo } \\
\text { demuestra } \\
\text { - Balbucea intencionalmente } \\
\text { para llamar la atención. } \\
\text { Sonrie a las caras. }\end{array}$ & $\begin{array}{l}\text { Puede distinguir los adultos } \\
\text { conocidos de los } \\
\text { desconocidos. } \\
\text { - Comienza a móstrar miedo } \\
\text { hacia los extraños. }\end{array}$ \\
\hline $\begin{array}{c}1 \text { a } 2 \text { ANOS } \\
\vdots \\
\because\end{array}$ & $\begin{array}{l}\text { - Se resiste a realizar algo } \\
\text { que no desea. } \\
\text { - Identifica partes del cuerpo. } \\
\text { Percibe la aprobación y } \\
\text { desaprobación de sus } \\
\text { actos. } \\
\text { - Obedece ordenes. } \\
\text { - Comprende el significado } \\
\text { YO. } \\
\text { Se siente culpable cuando } \\
\text { hace algo indebido. }\end{array}$ & $\begin{array}{l}\text { - Identifica a los miembros de } \\
\text { su familia nombrándolos o } \\
\text { señalándolos. } \\
\text { Demuestra sus afectos a los } \\
\text { conocidos mediante besos y } \\
\text { abrazos. } \\
\text { Demuestra tristeza cuando lo } \\
\text { dejan solo. }\end{array}$ & $\begin{array}{l}\text { - Se interesa por participar en } \\
\text { una interacción social. } \\
\text { Repite una acción si lo } \\
\text { aplauden. } \\
\because \\
\end{array}$ \\
\hline 2 A 3 ANOS & $\begin{array}{l}\text { Aprende a decir gracias } \\
\text { cuando recibe algo. } \\
\text { - Señala algunas partes del } \\
\text { cuerpo. } \\
\text { Expresa diferentes } \\
\text { emociones como alegría, } \\
\text { rabia o tristeza.. } \\
\text { - Expresa con gestos sus } \\
\text { estados de ánimo. } \\
\text { Quiere todo para el. }\end{array}$ & $\begin{array}{l}\text { Identifica a los miembros de } \\
\text { su familia nombrándolos o } \\
\text { señalándolos. } \\
\text { Demuestra sus afectos a los } \\
\text { conocidos mediante besos y } \\
\text { abrazios. } \\
\text { Demuestra tristeza cuando lo } \\
\text { dejan solo. } \\
\text { Trata de sobrepasar los límites } \\
\text { que le imponen sus padres. }\end{array}$ & $\begin{array}{l}\text { - Domina gradualmente su } \\
\text { ambiente. } \\
\text { - Le gusta estar acompañado. } \\
\text { - Comparte juguetes con otros } \\
\text { niños. } \\
\text { - Juega en forma paralela : al } \\
\text { lado de otros niños pero no } \\
\text { con ellos. } \\
\text { - Reconoce la expresión de } \\
\text { emociones en las otras } \\
\text { personas. }\end{array}$ \\
\hline
\end{tabular}


(Continuación)

\begin{tabular}{|c|c|c|c|}
\hline 3 a 4 ANOS & $\begin{array}{l}\text { - Aprende a comportarse de } \\
\text { acuerdo a la situación. } \\
\text { - Expresa sus deseos, } \\
\text { sentimientos y problemas } \\
\text { por medio de la palabra. } \\
\text { - Se siente seguro de lo que } \\
\text { realizá. } \\
\text { - Comienza proceso de } \\
\text { identificación sexual. } \\
\text { - Expresa limitaciones. } \\
\text { - Empieza a expresar } \\
\text { opiniones. } \\
\text { - Pide actuar por sí solo. }\end{array}$ & $\begin{array}{l}\text { - Atrae la atención del adulto. } \\
\text { - Identifica roles masculinos y } \\
\text { femeninos } \\
\text { - Manifiesta las actitudes de los } \\
\text { sexos en sus juegos. } \\
\text { - Tiene un amigo especial. }\end{array}$ & $\begin{array}{l}\text { - Sabe que esperan los demás. } \\
\text { - Se él. } \\
\text { - Pocomunica con los demás. } \\
\text { demás. } \\
\text { - Capta las expresiones } \\
\text { emocionäles de los otros. } \\
\text { - Colabora con los adultos. }\end{array}$ \\
\hline 4 a 5 AÑOS & $\begin{array}{l}\text { Dice su nombre completo } \\
\text { - Identifica su sexo } \\
\text { Hace dramatizaciones y } \\
\text { juegos para entender } e \\
\text { intentar satisfacer } \\
\text { necesidades. } \\
\text { - Empieza a formar los } \\
\text { primeros valores morales. }\end{array}$ & $\begin{array}{l}\text { Elige a sus amigos. } \\
\text { - Aprende a resolver las peleas } \\
\text { con palabras. } \\
\text { - Le gusta que lo feliciten los } \\
\text { adultos. }\end{array}$ & $\begin{array}{l}\text { - Utiliza con más frecuencia las } \\
\text { reglas de cortesía : dice por } \\
\text { favor o pide permiso } \\
\text { - Muestra interés por jugar con } \\
\text { otros niños. } \\
\text { - Respeta nomas en } \\
\text { actividades con otros niños. } \\
\text { - Se interesa por participar en } \\
\text { las actividades de los adultos. }\end{array}$ \\
\hline 5 a 6 AÑOS & $\begin{array}{l}\text { - Da explicaciones cuando se } \\
\text { le pregunta. } \\
\text { - Sabe comunicar lo que } \\
\text { desea. } \\
\text { - Le interesa satisfacer } \\
\text { primero sus deseos. } \\
\text { - Manifiesta sus emociones } \\
\text { - Le entristecen las historias. } \\
\text { - Tiene motivos más } \\
\text { subjetivos. } \\
\text { - Le gusta agradar a los } \\
\text { demás } \\
\text { - Soluciona problemas solo. } \\
\text { - Entiende las normas } \\
\text { sencillas }\end{array}$ & $\begin{array}{l}\text { - Explica a otro las reglas de un } \\
\text { juego o actividad. } \\
\text { - Está aprendiendo a dar y } \\
\text { recibir. } \\
\text { - Es más consciente del otro y } \\
\text { de su relación con él. } \\
\text { - Expresa con mayor frecuencia } \\
\text { su afecto. }\end{array}$ & $\begin{array}{l}\text { - Tiene más de un amigo } \\
\text { - Participa en juegos } \\
\text { - Interactitivos. } \\
\text { diversos grupos. } \\
\text { - Le encantan los juegos } \\
\text { sociales y de roles. } \\
\text { - Se preocupa por los demás } \\
\text { - Explora a través del lenguaje } \\
\text { las actividades de los adultos. } \\
\text { - Reconoce su pertenencia a } \\
\text { grupos sociales. }\end{array}$ \\
\hline
\end{tabular}

La razón por la cual es importante crear programas de desarrollo emocional en todos los jardines y colegios de Colombia, es porque esta en manos, de los educadores, ayudar a jóvenes y niños a:

- A formar la autoestima: ya que el autoconocimiento, la aceptación y la autoconfianza, son indispensables para enfrentar las situaciones de la vida.

- A conocer al otro: al hermano, al amigo o al vecino. Pues la convivencia empieza por salir de uno mismo y reconocer a los demás. 
- A ser una persona solidaria: con deberes y derechos sociales. Capaz de asumir un lugar en la comunidad y conciente de la responsabilidad de hacerlo.

- Acrecer con habilidades para: conciliar, comprender, perdonar, tomar decisiones y respetar las diferencias. Dar a los demás, no solo cosas materiales sino calor humano.

\section{Marco teórico}

El programa se ha fundamentado en los planteamientos de los siguientes autores: Los siquiatras Luis Carlos Restrepo y Daniel Goleman. Las sicólogas Ana María León, Lucia Pinto y Martha Rodríguez.

El siquiatra Luis Carlos Restrepo (2) en su libro "El derecho a la ternura" plantea que: La cultura actual se ha vuelto un enemigo de las personas, un ejemplo de esto es que nos hemos vuelto prevenidos con los extraños pues pensamos que nos van a hacer daño.

Además dice que la vida pública de las personas se construye dentro de la vida privada de la familia. Por tanto el núcleo familiar parece estar favoreciendo relaciones carentes de afecto. Esta situación influye tanto en la prevención frente a los extraños como en las relaciones entre padres e hijos.

Luis Carlos Restrepo propone que es necesario enseñar a las personas a desarrollar la capacidad de sentir el afecto y de demostrarlo. Por ejemplo hay que permitirle a los hombres llorar y no negarles el derecho a sentir, por el hecho de ser hombres. Con esta propuesta el autor insiste en que las personas deben integrar el corazón y la razón en sus acciones. También plantea que las personas pueden desarrollar la capacidad de dar y recibir ternura, de construir lazos cálidos y de vivir la intimidad.

Daniel Goleman (1), siquiatra norteamericano, en su libro "La Inteligencia Emocional" presenta la inteligencia emocional como un elemento importante para que las personas tengan éxito, ya que tener un coeficiente intelectual alto por sí solo, no es garantía. El autor, retomando los estudios de Howard Gardner sobre inteligencias múltiples, presenta dos tipos de inteligencia emocional: La intra-personal o el conocimiento de sí mismo, y la inter-personal o el conocimiento de los demás. El 
desarrollo equilibrado de estas inteligencias, permite la interacción efectiva y acertada del individuo con su entorno.

Göleman también plantea que las personas nacen con una base genética y orgánica para desarrollar la inteligencia emocional. Existen en el código genético, modelos para reaccionar ante las situaciones, que se ven influidos por funciones neurológicas como los reflejos.

El psiquiatra expone en su libro cómo la Amígdala, una estructura del Sistema Nervioso Central que se encuentra sobre el Tallo Cerebral y cerca àl Sistema Límbico, responde à los estímulos emocionales antes de que la Corteza lo haga para interpretar la situación. La Amígdala también almacena la Memoria Emocional, la cual determina la manera de reaccionar de las personas.

El comportamiento de las personas depende de su estabilidad emocional y su capacidad de desarrollar autocontrol. El planteamiento básico; novedoso y esperanzador de Goleman, es que el autocontrol y la estabilidad pueden enseñarse, ya que la maduración neurológica de los centros que controlan estas habilidades, se demora más o menos hasta los 16 años y se desarrolla gracias a los estímulos ambientales. Por lo tanto el aprendizaje de las habilidades anteriormente anotadas, se puede favorecer $y / o$ modificar a través de experiencias sistemáticas durante la niñez.

A raíz de estos planteamientos, Goleman propone incluir dentro del programa escolar, un curriculum para las emociones, no dejando que se desarrollen al azar, sino ayudando a formarlas, a reconocerlas y a saberlas expresar y manejar. Estos programas pueden actuar a largo plazo, como preventivos y correctivos para que más niños y jóvenes desarrollen una vida emocional más sana.

En Estados Unidos hay algunos colegios que ya trabajan una asignatura que llaman Self-science (Ciencia del autoconocimiento). En ella se incluye por ejemplo, la lectura de claves para la interacción social. Esta lectura de claves permite entender los estados de ánimo y las intenciones de las personas, a través de sus gestos. Otros elementos del programa son: cómo tomar decisiones personales, desarrollar estrategias de comunicación y solución de conflictos. 
Ana María León (6), sicóloga peruana, plantea la necesidad de reconstruir la escala de valores con que se maneja la ética actual. Según ella, ésta se ha venido invirtiendo en los últimos años. Por ejemplo, el respeto o la justicia se han colocado por debajo del ansia de poder y dinero.

Ella define al hombre moderno como una persona egocéntrica, que actúa sólo por propia conveniencia y cuya ética gira alrededor del placer. Lo denomina "el hombre light", quien quiere ser apuesto, usar ropa de marca y estar en la cina del mundo, sin importar a quien aplaste para lograrlo.

La autora considera que el hijo de este hombre, esta copiando el modelo de su padre y vive en conflicto. El niño se debate entre el ejemplo de su familia y lo que pretende enseñarle el colegio.

Ana María León también explica que los maestros están asumiendo nuevos roles, al tomar la responsabilidad de iniciar a los niños en la creación de una adecuada escala de valores. Función que también es responsabilidad de la familia.

Para dar solución a esta situación, la autora hace énfasis en la necesidad de reforzar en los padres la tarea de ser educadores morales. Esta tarea debe partir de una redimensión de la escala de valores. Se necesita, por ejemplo, enseñar al niño a desarrollar el respeto por sí mismo, por el otro y por el ambiente que le rodea; a asumir responsabilidades para consigo mismo y los demás, y a tener una actitud equilibrada entre su libertad y la de los otros. Para lograrlo se proponen consejos tan antiguos como dar buen ejemplo, pues una acción vale más que mil palabras; enseñar hablando, ya que se debe predicar lo que se hace; e inculcar el amor amando, pues se aprende más fácil aquello que se vivencia.

En los aportes de Lucia Pinto (7) y Martha Rodríguez, (5) se plantea la urgencia de enseñar al niño a formar su vida ética y social.

Estas personas, como los anteriores autores, fundamentan su trabajo en el hecho de que la sabiduría es el resultado del intelecto y el sentimiento. Es decir, que el hombre no puede separar su afecto de sus acciones si desea ser una persona íntegra, capaz y feliz. 
Las autoras plantean que la vida afectiva se organiza en función de valores y principios de cooperación social. Esto debido a que el hombre vive en comunidad, y construye su realidad reconociendo la existencia del otro.

Además describen a la Escuela como el primer espacio de actuación pública del niño. El lugar donde aprende a interactuar con los demás desde su vida privada. Por esta razón, dan gran importancia a la construcción del sentido social en el niño, a partir de las experiencias escolares.

En esta propuesta, la acción educativa se dirige a padres, niños y medio social, trabajando en tres dimensiones: La formación afectiva del individuo para favorecer su interacción social; una educación sexual positiva y el desarrollo de la responsabilidad social.

El método que se utiliza es enseñar a las personas a "saber hacer". Es decir, ensenarles a hacer las cosas para ser más dueños de sí mismos, capaces de desenvolverse en el medio que viven y dispuestos a actuar con una orientación ética y social.

\section{Programa de Desarrollo Emocional}

El programa de Desarrollo Emocional del jardín infantil por un mañana utiliza estrategias que favorecen el desarrollo del autoconocimiento y la autoestima, el conocimiento del otro, y el sentido de grupo como base de la competencia social.

El programa se diseño siguiendo las pautas del Modelo de Pedagogía Participativa (Amaya, Lizarazo, y otros, 1997), el cuál se centra en el niño, aplicando acciones educativas que integran los saberes de la Pedagogía, la Terapia Ocupacional y la Fonoaudiología. El modelo tiene objetivos interactivos de estimulación, prevención, evaluación y atención de los procesos básicos de desarrollo y da gran importancia a la participación de la familia como multiplicador del proceso educativo.

\section{Objetivos del programa}

La meta del programa es educar niños felices consigo mismos, que inicien la construcción de su escala de valores y ayuden a formar una sociedad más humana 
basada en el respeto, el derecho a expresar sentimientos, la tolerancia y la solidaridad.

Los objetivos de programa son:

- Facilitar y hacer amable al niño, el paso de la casa al colegio o sea de la vida privada a la pública.

- Desarrollar el conocimiento personal, trabajando de la mano la autoestima.

- Propiciar al niño el conocimiento del otro, para que aprenda a interactuar con los demás.

- Favorecer el sentido de grupo para enseñar al niño a trabajar en equipo.

- Enseñar a actuar democráticamente para desarrollar principios de cooperación social.

\section{Estrategias del Programa de Desarrollo Emocional}

Las estrategias para cumplir con los objetivos son las siguientes: Facilitar y hacer amable el paso de la casa al colegio; Desarrollar el conocimiento personal, el conocimiento del otro, el sentido de grupo y la actuación democrática, y elaborar una cartilla de trabajo para el niño, el maestro y la familia. A continuación se encuentran las actividades que se llevan a cabo en la cotidianeidad del Jardín.

\section{Facilitar y hacer amable el paso de la casa al colegio}

- Se prepara el primer acercamiento del maestro al niño. Por ejemplo, el maestro puede ir a visitar al niño a su casa antes del primer día de colegio, con el fin de presentarse y conocerlo. También puede citar a todos los niños nuevos unos días antes de iniciar las clases para conozcan el salón y se familiaricen con él.

- Se facilita la presentación del niño ante el grupo, por medio de una ficha en la que se escriben las principales características de este.

- Se favorece la adaptación del niño al colegio a través de consejos prácticos dirigidos a la familia para que ayuden en el proceso. También se da gran importancia a la actitud del maestro ante el niño ya que ésta es la conexión entre la casa y el colegio.

- Se inculca el sentido de pertenencia en grupo por medio de la utilización de distintivos que caracterizan cada grupo. 


\section{Desarrollar el conocimiento personal, el conocimiento del otro, el sen-} tido de grupo y la actuación democrática: se utilizan .las siguientes estrategias: La actitud de vida del maestro; Una cartilla de trabajo para el niño, el maestro y la familia; Un programa de personaje de la semana; una serie de actividades comunitarias que favorecen la participación en grupo; un conjunto de planes caseros diseñados para enriquecer el proceso individual; una serie de tarjetas de cortesía que contienen mensajes de apoyo para diversas ocasiones; una biblioteca con cuentos y canciones. Las estrategias se ejecutan dentro de la vida diaria del jardín:

- Un maestro cuya actitud de vida lo compromete a:

- Conducir a apoyar las expresiones emocionales dentro de su clase.

- Dar ejemplo con su comportamiento.

- Demostrar cómo se hacen las cosas.

- Identificar la oportunidad de enseñar hablando.

- Desarrollar la habilidad de trasmitir sentimientos, reglas, costumbres y estilos para hacer las cosas.

- Tener la capacidad de formar y fortalecer los valores humanos.

- Una cartilla de trabajo para el niño, el maestro y la familia, que es la puesta en el papel y lápiz del programa. Esta cartilla contiene los siguientes temas: conocimiento personal, conocimiento del otro, sentido de grupo y actuación democrática.

La cartilla también tienen preparadas actividades para situaciones especiales y de crisis. Por ejemplo, la separación de los padres, el nacimiento de un hermanito, la enfermedad de un familiar o la muerte. Estas actividades se realizan tanto en el colegio como en la casa.

- Un programa de personaje de la semana que consiste en que cada niño del curso es muy importante durante una semana. El niño trae una cartelera con un tema específico según el nivel.

Los temas son los siguientes: así soy yo, yo he crecido, la historia de mi vida, lo que me gusta hacer, cuéntenme por que me quieren, las cosas que ya se hacer sólo. Durante esta semana el niño tiene funciones especiales, las cuales le asigna la profesora, haciéndolo muy importante.

- Una serie de actividades comunitarias que favorecen la participación en grupo. Para esto se programan las semanas y días especiales: semana de 
valores, semana cultural, visita de los padres, elecciones infantiles, día de la naturaleza, etc.

- Un conjunto de planes caseros diseñados para enriquecer el proceso individual dándole a los padres actividades y consejos prácticos. De esta manera la familia participa activamente.

- Una serie de tarjetas de cortesía que contienen mensajes de apoyo para diversas ocasiones. Por ejemplo, cumpleaños, enfermedad, nacimiento de un hermanito, duelo. Otra serie dé tarjetas dirigidas a motivar las actitudes positivas de los niños. Por ejemplo, progresos, buenas noticias, continuaasí.

- Una biblioteca con cuentos y canciones que le permiten al niño expresar sentimientos, clarificar estados de ánimo y comprender situaciones. Además estas actividades le dan ideas a los niños para actuar en ciertas ocasilones y le dejan un mensaje.

El éxito de un programa de desarrollo emocional radica en las personas, ya que la responsabilidad de llevarlo adelante es compartida entre la familia y el colegio. Con la decidida colaboración y compromiso de padres, niños y maestros, las actividades del jardín infantil darán los resultados esperados.

El importante papel de quien educa se puede resumir en el pensamiento de Olegario G. de Cardedal:

"Un educador es esencialmente un creyente y un poeta. Si no ofreciese crédito a la materia viva que tiene ante sus ojos, no le dirigiría la palabra para hacer nacer al hombre viviente; y si no fuese un poeta, es decir, un creador, no accedería a esa libertad virgen, frágil y bella como un cristal de roca".

Olegario G. de Cardedal 


\section{BIBLIOGRAFÍA}

1. Goleman, Daniel. (1995). Inteligencia emocional. Bantam Ed. New York.

2. Restrepo, Luis Carlos. (1994). El derecho a la ternura. Arango Editores. Santafé de Bogotá.

3. Restrepo, Luis Carlos (1994). Ecología Humana: Una propuesta de reconstrucción cultural desde la ternura. Pontificia Universidad Javeriana. . Facultad de Psicología, programa de educación continuada. Santafé de Bogotá.

4. Shapiro, Lawrence (1997). La Inteligencia Emocional de los niños. Vergara Editor. Buenos Aires. Cano Formas e impresos. Colombia.

5. Rodríguez, Martha (1997). La escuela: El primer espacio de actuación pública del niño. Criterios y observaciones en la formación de Hábitos ciudadanos. Fundación Social. Santafé de Bogotá.

6. León, Ana María (1997). La formación de Valores éticos en los niños. En: Primer Seminario sobre Constructivismo en Educación Inicial. Lima, Perú.

7. Pinto, Lucía (1990). La formación de la afectividad. Programa Escuela de Padres. Federación de asociaciones de padres de los Colegios de la Compañía de Jesús. Santafé de Bogotá.

8. Amaya, Mariluces; Lizarazo, R.; Restrepo, B.; Tobón, M.; Trujillo, C. Modelo de Pedagogía Participativa para Programas de Educación Preescolar. Asociación Colombiana de Terapia Ocupacional, (Editores), Revista Ocupación Humana. Volúmen 6, número 4 de 1996. Santafé de Bogotá. 


\section{EMOTIONAL DEVELOPMENT PROGRAM Abstract}

The great amount of selfishess, lack of values and great viloence that you find in the world today, has led several authors to write about the influence that emotional developmente has in people's life. At Por un Mañana preschool we have beenn working in implementing an Emotional Development Progran specially for the preschool years knowing how important this subject is and how it is a great necessity in our country.

The progran was developed fallowing the theory of Participative Pedagogy. (Amaya, Lizarazo and others, 1997).

This model is centered around the child and it integrates the knowledge of the three different bases: Pedagogy, lannguage and occupational therapies. Therefore Participative pedagogy applies aducational actions in an ecclective way.

The program is intended for very small children between the ages of one and $a$ half and five years. However it is considered that the basic guidelines are valid for older children as well.

The program has been based in the theories of two psychiatricians Doctor $\mathrm{Da}$ niel Goleman from U.S.A: and doctor Luis Carlos Restrepo from Colombia. And several psycoligsts: Ana María León from Perú, Lucia Pinto y Martha Rodríguez Fom Colombia.

The Program main objectives are to develop self awarness, to encourage an propiciate the undertanding of others and the groupe and to teach how to act democratically.

Key words: Emotional Development, human values, self awareness, social cooperation, undertanding of others, self knowledge, self steem. 